\title{
Clip retraction of the tentorium: application of a novel technique for tentorial retraction during supracerebellar transtentorial approaches
}

\author{
*Jacob F. Baranoski, MD, Ankush Bajaj, BS, Colin J. Przybylowski, MD, Joshua S. Catapano, MD, \\ Fabio A. Frisoli, MD, Michael J. Lang, MD, and Michael T. Lawton, MD
}

Department of Neurosurgery, Barrow Neurological Institute, St. Joseph's Hospital and Medical Center, Phoenix, Arizona

Supracerebellar transtentorial (SCTT) approaches have become a popular option for treatment of a variety of pathologies in the medial and basal temporal and occipital lobes and thalamus. Transtentorial approaches provide numerous advantages over transcortical approaches, including obviating the need to traverse eloquent cortex, not requiring parenchymal retraction, and circumventing critical vascular structures. All of these approaches require a tentorial opening, and numerous techniques for retraction of the incised tentorium have been described, including sutures, fixed retractors, and electrocautery. However, all of these techniques have considerable drawbacks and limitations. The authors describe a novel application of clip retraction of the tentorium to the supracerebellar approaches in which an aneurysm clip is used to suspend the tentorial flap, and an illustrative case is provided. Clip retraction of the tentorium is an efficient, straightforward adaptation of an established technique, typically used for subtemporal approaches, that improves visualization and surgical ergonomics with little risk to nearby venous structures. The authors find this technique particularly useful for the contralateral SCTT approaches.

https://thejns.org/doi/abs/10.3171/2020.2.JNS192952

KEYWORDS cavernous malformation; supracerebellar infratentorial approach; supracerebellar transtentorial approach; surgical technique; tentorium; vascular disorders

$\mathrm{T}$ HE tentorium is a free dural structure that can be incised and retracted to expand exposures of deep medial anatomical regions. Subtemporal approaches to the basilar bifurcation or trunk for aneurysm clipping employ this technique routinely, incising the tentorium posterior to the entry of the trochlear nerve into its dural sleeve and connecting the middle and posterior cranial compartments. ${ }^{1,2}$ The posterior tentorial leaflet is typically reflected laterally and held in place with a stay suture. Hernesniemi et al. ${ }^{3}$ introduced a clever technique that simplified this maneuver, making a second incision in the tentorium laterally and using an aneurysm clip to hold the folded leaflet in the blades of the clip, with one blade passing through the tentorial incision. This "clip retraction" technique was widely accepted as being easier and quicker than the use of sutures and is now almost a standard step in the subtemporal approach.

Recently, supracerebellar transtentorial (SCTT) approaches have become a popular option for treatment of a variety of pathologic conditions in the medial and basal temporal and occipital lobes. ${ }^{4-6}$ Lesions such as meningiomas, ${ }^{7}$ gliomas, cavernous malformations $(\mathrm{CMs}),{ }^{8-10}$ and arteriovenous malformations ${ }^{11,12}$ have been removed through posterior approaches through the tentorium, often with the use of the sitting position and gravity retraction of the cerebellum to widen the supracerebellar space and extend the upward reach beyond the tentorium. We and others have found this strategy useful for the treatment of thalamic pathologic entities that abut the posterior-inferior surface of the pulvinar. ${ }^{8,10,13,14}$ Transtentorial approaches

ABBREVIATIONS CM = cavernous malformation; PCA = posterior cerebral artery; SCTT = supracerebellar transtentorial. SUBMITTED October 30, 2019. ACCEPTED February 4, 2020.

* J.F.B. and A.B. contributed equally to this work. 


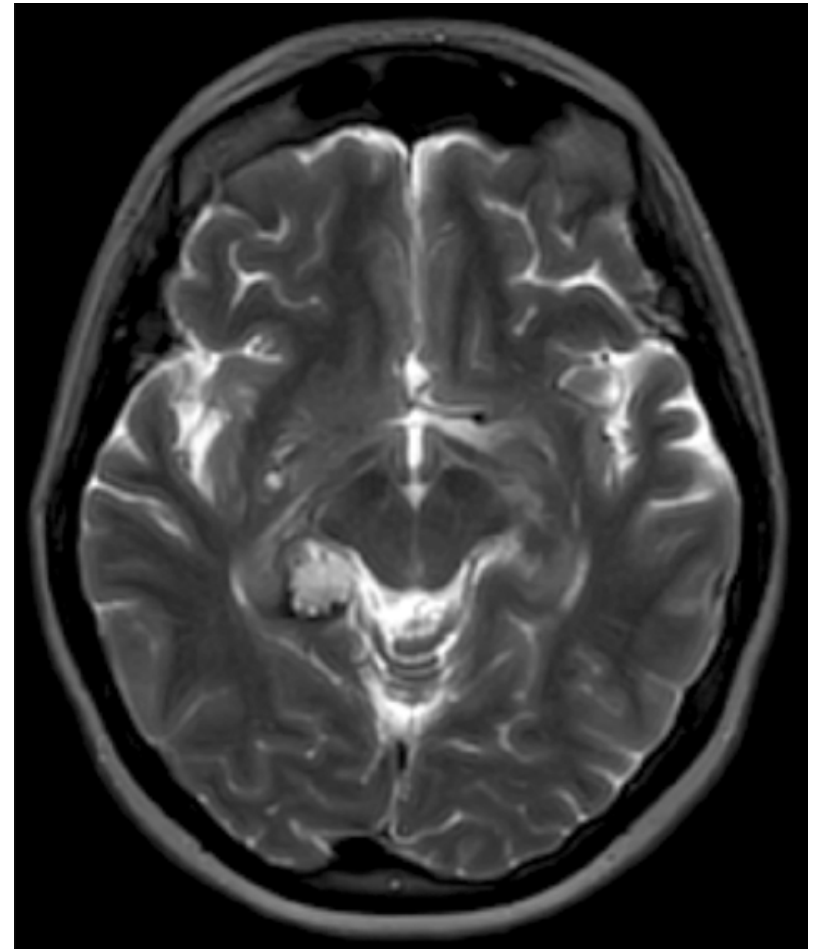

FIG. 1. Preoperative axial T2-weighted MR image showing a CM located in the right posterior medial temporal lobe.

obviate the need to traverse eloquent cortex, which makes these approaches preferable for the treatment of parenchymal lesions in the dominant hemisphere. Transtentorial approaches also circumvent key vascular structures, such as the vein of Labbé.

By definition, transtentorial approaches require a tentorial opening to reach their target lesion..$^{8,9,15}$ Numerous techniques for retraction of the incised tentorium have been described, including sutures, fixed retractors, and electrocautery. ${ }^{4}$ However, to our knowledge, no one has adapted the technique described by Hernesniemi et al. ${ }^{3}$ for obtaining subtemporal exposures to attaining SCTT exposures. In this paper, we describe the novel application of clip retraction of the tentorium to obtain a supracerebellar approach in a case of a right posterior medial temporal lobe $\mathrm{CM}$ resection. We find this ergonomic technique particularly useful with the contralateral SCTT approach, in which the lateral exposure is more extreme than with the ipsilateral SCTT approach. ${ }^{9}$

\section{Case Presentation and Technical Note History and Examination}

A 65-year-old woman presented with seizures that were recurrent despite the administration of maximal medical therapy. Diagnostic imaging findings indicated a nonenhancing lesion in the right posterior medial temporal lobe that was $1.5 \mathrm{~cm}$ in diameter, consistent with a CM (Fig. 1). Because of the patient's medically refractory seizures, microsurgical resection of the CM was recommended.
With use of the two-point method,,$^{13}$ we determined that the location of the lesion was ideal for a contralateral SCTT approach, with the patient in the sitting position. ${ }^{9}$ After an appropriate preoperative workup that included an echocardiogram with a bubble study, patient consent was obtained, and the patient was scheduled for surgery.

\section{Operative Technique}

The patient was placed in the sitting position, a midline suboccipital incision was made, and a suboccipital craniotomy eccentric to the left side was performed that exposed the left transverse sinus and the edge of the left torcula. The dura was opened and flapped superiorly, which placed gentle traction on the left transverse sinus and exposed the supracerebellar infratentorial corridor. Cerebrospinal fluid drainage and the gravity retraction afforded by the sitting position allowed for ample relaxation of the cerebellum. Arachnoid dissection with preservation of the cerebellar veins allowed for further widening of the supracerebellar infratentorial corridor. Dissection was continued anteriorly to the quadrigeminal cistern, which exposed the posterior cerebral artery (PCA), trochlear nerve, and vein of Galen. Intraoperative neuronavigation identified the necessary trajectory to the right posterior medial temporal lobe $\mathrm{CM}$ through the tentorium, and a corresponding tentorial flap was planned. The tentorium was sharply transected transversely (Fig. 2A). A separate small incision was made in the tentorial dura laterally to accommodate one blade of the aneurysm clip. The posterior leaflet of incised tentorium was folded laterally, and a straight aneurysm clip was used to retract the leaflet. One of the clip's blades was inserted through the lateral tentorial incision, and the other blade held the tentorial flap against the tentorium. This maneuver suspended the tentorial flap to enhance visualization and operative maneuverability within the surgical corridor (Fig. 2B-D). This maneuver was faster and simpler than securing the tentorial flap with a deep stitch in a relatively tight corridor.

With the tentorium incised and the flap clipped to the adjacent tentorium, the CM was easily identified in the right posterior medial temporal lobe, with its associated hemosiderin staining of the parenchyma. The $\mathrm{CM}$ was circumferentially dissected and was resected in its entirety. After resection, the aneurysm clip was removed to free the tentorial flap. A watertight dural closure was performed, and the remainder of the case proceeded in a standard fashion.

\section{Postoperative Course}

The patient tolerated the procedure very well. Postoperative imaging confirmed complete resection of the CM (Fig. 3). At her 6-week follow-up examination, the patient had made a complete recovery and exhibited no neurological deficits.

\section{Discussion}

Because of their advantages over transcortical approaches, SCTT approaches are becoming more common for the treatment of pathologic conditions in the posterior thalamus and medial temporooccipital space. Previous 

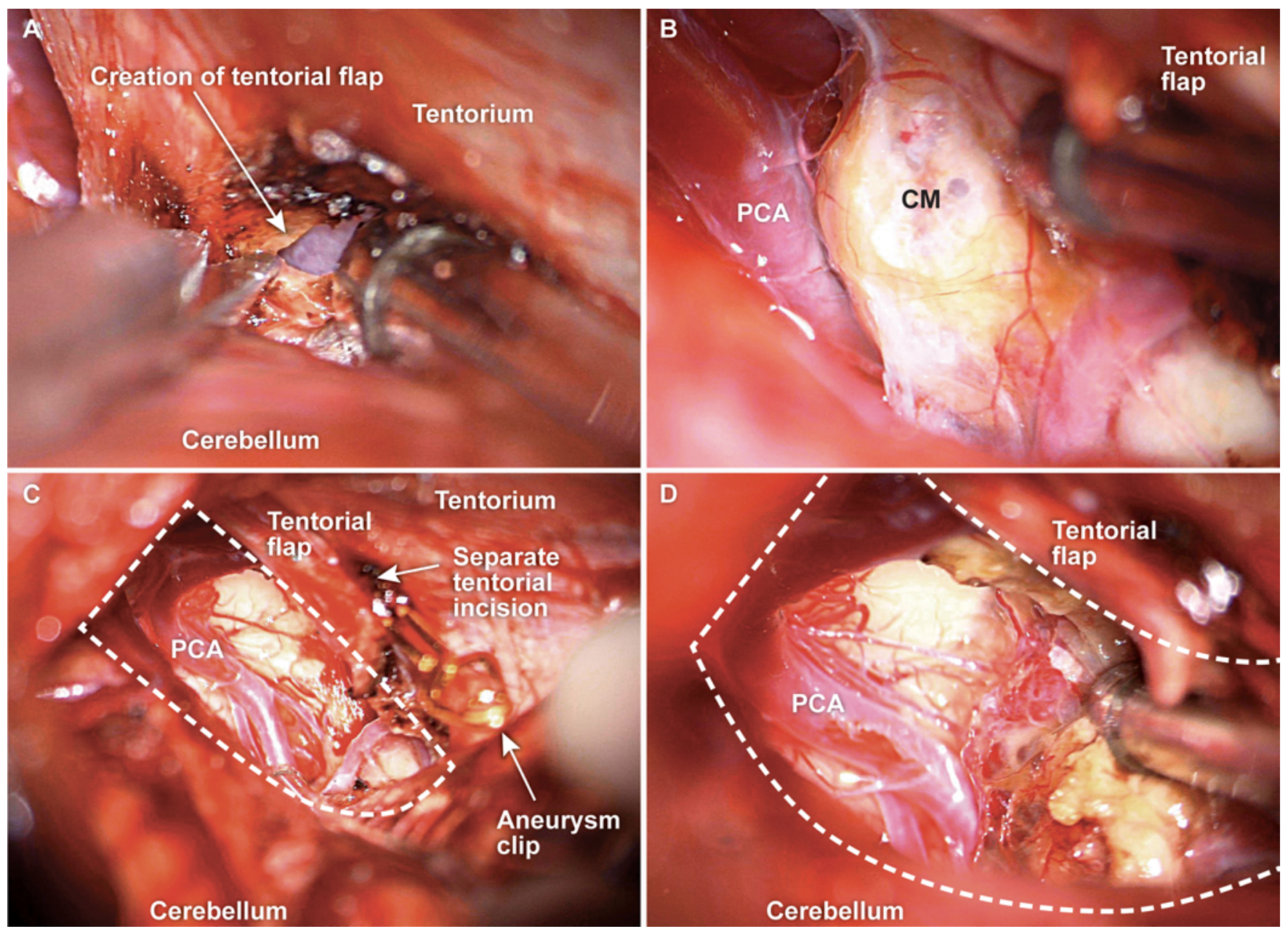

ABOVE

FIG. 2. Intraoperative photographs showing (A) the sharp opening of the tentorium to create the tentorial flap, (B) exposure of the CM after clip retraction of the tentorium, and $(\mathbf{C})$ the surgical corridor (dashed outline) afforded by the clip retraction technique, which provides excellent visualization of the lesions as well as surgical ergonomics. Note the straight aneurysm clip with one of the clip's blades inserted through a separate tentorial incision and the other blade securing the tentorial flap against the tentorium. D: A higher magnification view of the surgical corridor (dashed outline) is also shown. Used with permission from Barrow Neurological Institute, Phoenix, Arizona.

\section{RIGHT}

FIG. 3. Postoperative axial T2-weighted MR image showing complete resection of the CM.

studies have characterized the use of either SCTT or occipital transtentorial approaches for the microsurgical resection of meningiomas, ${ }^{4,6,16} \mathrm{CMs},{ }^{8-10}$ epidermoid cysts, ${ }^{17}$ and other pathologic conditions. ${ }^{11,12,18,19}$ Compared with transcortical routes, transtentorial approaches provide excellent anatomical exposure without traversal of eloquent cortex or retraction of parenchyma. However, a limitation of these transtentorial approaches is the narrow corridor through the tentorium, which may hinder visualization or limit the dexterity of the surgeon. The clip retraction

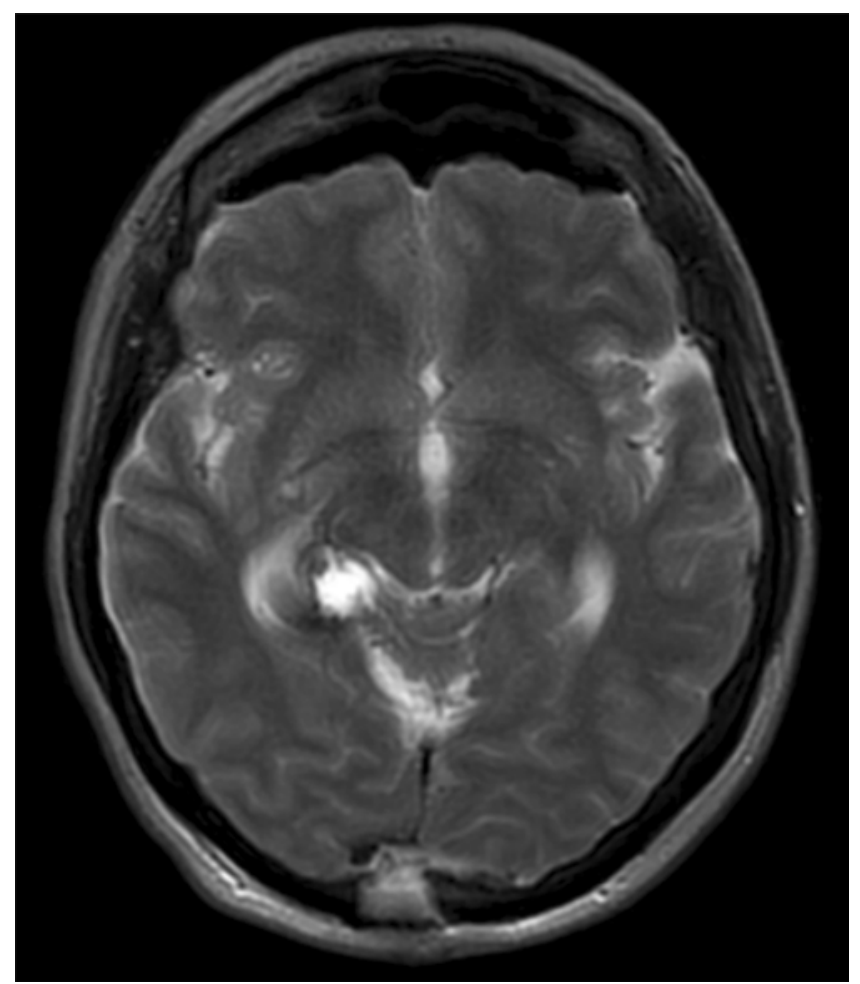




\section{A}

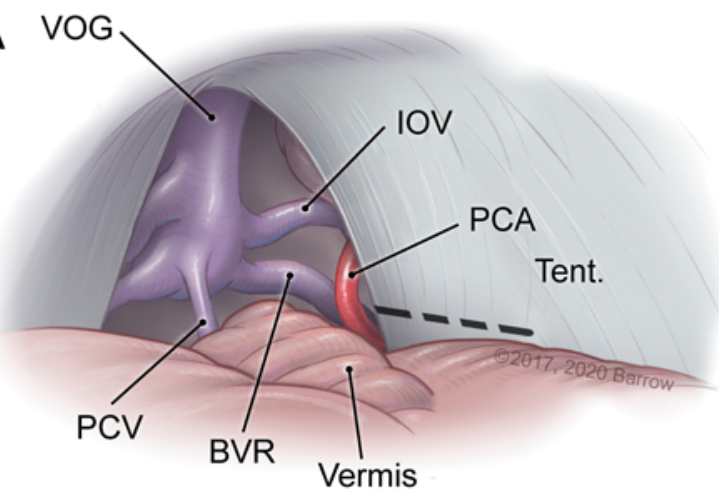

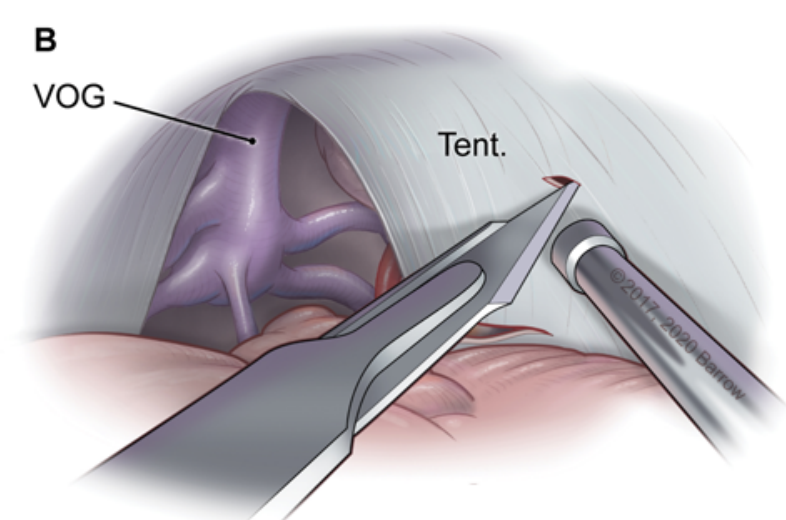

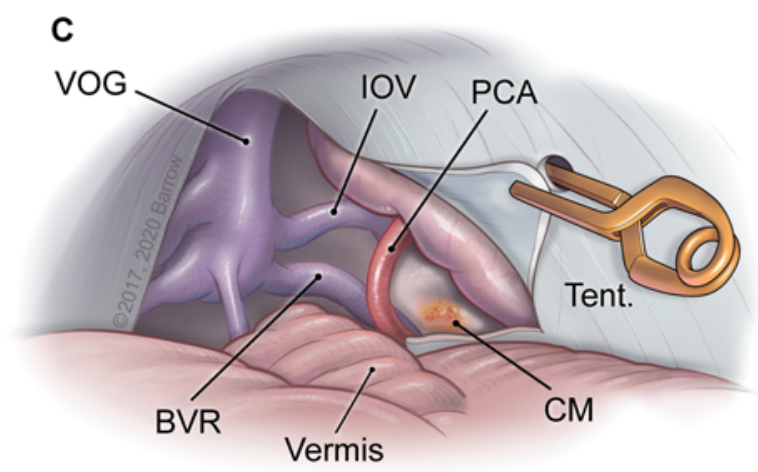

FIG. 4. Illustrations of the clip retraction technique for securing the tentorium with the use of a straight aneurysm clip, demonstrating $(\mathbf{A})$ the location of the tentorial cut (dashed line), (B) the separate incision made in the tentorium to accommodate the blade of the clip, and $(\mathbf{C})$ the placement of the clip retracting the tentorial flap against the tentorium and the exposure that this technique affords. $B V R=$ basal vein of Rosenthal; $I O V=$ internal occipital vein; $P C V=$ precentral cerebellar vein; Tent. = tentorium; VOG = vein of Galen. Used with permission from Barrow Neurological Institute, Phoenix, Arizona.

technique described herein quickly and easily secures the tentorial flap and prevents the flap from obscuring the surgical field, thereby improving the SCTT approach (Fig. 4).

Conventional techniques for mobilizing the incised tentorium include the use of sutures, fixed retractors, electrocautery, or large flaps. Stitching the tentorium at this depth and with the patient in the sitting position can be cumbersome, and retractors obscure the surgical field. Other neurosurgeons have offered alternatives to tentorial flap suspension. In 2012, de Oliveira et al. ${ }^{20}$ published a series of SCTT approaches in which they completely resected a portion of the tentorium, as opposed to creating a flap. Although this technique works well, it also has considerable drawbacks that include the need for additional tentorial incisions, management of bleeding from tentorial sinuses, injury to tentorial draining veins, and morbidity associated with venous occlusion.

Clip retraction of the tentorium is an efficient, straightforward adaptation of an established technique typically used for subtemporal approaches that incise the tentorium. Clip retraction improves visualization and surgical ergonomics with little risk to nearby venous structures. Elsewhere, we have reported our use of aneurysm clips to anchor slings to dural sleeves in the clivus for macrovascular decompressions of the vertebral and basilar arter- ies. ${ }^{21}$ This posterior clip retraction of the tentorium may be viewed as another example of the utility of aneurysm clips anchored to the dura to mobilize and hold obstructive anatomical features or compressive pathologic entities.

\section{Acknowledgments}

We thank the staff of Neuroscience Publications at Barrow Neurological Institute for assistance with manuscript preparation.

\section{References}

1. Tayebi Meybodi A, Benet A, Rodriguez Rubio R, et al. Comparative analysis of orbitozygomatic and subtemporal approaches to the basilar apex: a cadaveric study. World Neurosurg. 2018;119:e607-e616.

2. Tjahjadi M, Kivelev J, Serrone JC, et al. Factors determining surgical approaches to basilar bifurcation aneurysms and its surgical outcomes. Neurosurgery. 2016;78(2):181-191.

3. Hernesniemi J, Ishii K, Karatas A, et al. Surgical technique to retract the tentorial edge during subtemporal approach: technical note. Neurosurgery. 2005;57(4)(suppl):E408.

4. Golub D, Mehan ND, Kwan K, et al. Supracerebellar transtentorial approach for occipital meningioma to maximize visual preservation: technical note. Oper Neurosurg ( $\mathrm{Ha}$ gerstown). 2019;17(4):E177-E183.

5. Sun Q, Zhao X, Gandhi S, et al. Quantitative analysis of ipsilateral and contralateral supracerebellar infratentorial and occipital transtentorial approaches to the cisternal pulvinar: 
laboratory anatomical investigation [published online August 2, 2019]. J Neurosurg. doi:10.3171/2019.4.JNS19351

6. Zhao X, Belykh E, Przybylowski CJ, et al. Surgical treatment of falcotentorial meningiomas: a retrospective review of a single-institution experience [published online August 2, 2019]. J Neurosurg. doi:10.3171/2019.4.JNS19208

7. Watanabe T, Katayama Y, Fukushima T, Kawamata T. Lateral supracerebellar transtentorial approach for petroclival meningiomas: operative technique and outcome. J Neurosurg. 2011;115(1):49-54.

8. Iwami K, Fujii M, Saito K. Occipital transtentorial/falcine approach, a "cross-court" trajectory to accessing contralateral posterior thalamic lesions: case report. J Neurosurg. 2017;127(1):165-170.

9. Mascitelli J, Burkhardt JK, Gandhi S, Lawton MT. Contralateral supracerebellar-infratentorial approach for resection of thalamic cavernous malformations. Oper Neurosurg ( $\mathrm{Ha}$ gerstown). 2018;15(4):404-411.

10. Sarris CE, Atwal GS, Nakaji P. Thalamic cavernous malformations. Handb Clin Neurol. 2017;143:297-302.

11. Chaddad-Neto F, da Costa MDS, Caramanti RL, et al. Transtentorial approach for parahippocampal gyrus arteriovenous malformation resection: 3-dimensional operative video. Oper Neurosurg (Hagerstown). 2019;16(3):E83-E84.

12. Harput MV, Türe U. The paramedian supracerebellar-transtentorial approach to remove a posterior fusiform gyrus arteriovenous malformation. Neurosurg Focus. 2017;43(Video Suppl 1):V7.

13. Brown AP, Thompson BG, Spetzler RF: The two-point method: evaluating brain stem lesions. BNI Q. 1996;12(1):20-24.

14. Choudhri O, Davies J, Lawton MT. The supracerebellartranstentorial approach to vascular lesions in the inferomedial temporal lobe: 3-dimensional operative video. Oper Neurosurg (Hagerstown). 2017;13(4):536.

15. Kalani MY, Lei T, Martirosyan NL, Oppenlander ME, Spetzler RF, Nakaji P: Endoscope-assisted supracerebellar transtentorial approach to the posterior medial temporal lobe for resection of cavernous malformation. Neurosurg Focus. 2016;40(Video Suppl 1):1.

16. Nanda A, Patra DP, Savardekar A, et al. Tentorial meningiomas: reappraisal of surgical approaches and their outcomes. World Neurosurg. 2018;110:e177-e196.
17. Goel A, Shah A. Lateral supracerebellar transtentorial approach to a middle fossa epidermoid tumor. J Clin Neurosci. 2010;17(3):372-373.

18. Weil AG, Middleton AL, Niazi TN, et al. The supracerebellar-transtentorial approach to posteromedial temporal lesions in children with refractory epilepsy. J Neurosurg Pediatr. 2015;15(1):45-54.

19. Xinrui L, Sato Y, Dan M, et al. Total resection of brainstem extension of tentorial schwannoma using an occipital transtentorial approach. World Neurosurg. 2017;98:879.e13-879. e16.

20. de Oliveira JG, Párraga RG, Chaddad-Neto F, et al. Supracerebellar transtentorial approach-resection of the tentorium instead of an opening-to provide broad exposure of the mediobasal temporal lobe: anatomical aspects and surgical applications: clinical article. J Neurosurg. 2012;116(4):764-772.

21. Choudhri O, Connolly ID, Lawton MT. Macrovascular decompression of the brainstem and cranial nerves: evolution of an anteromedial vertebrobasilar artery transposition technique. Neurosurgery. 2017;81(2):367-376.

\section{Disclosures}

The authors report no conflict of interest concerning the materials or methods used in this study or the findings specified in this paper.

\section{Author Contributions}

Conception and design: Lawton, Baranoski. Acquisition of data: all authors. Analysis and interpretation of data: all authors. Drafting the article: Baranoski, Bajaj. Critically revising the article: all authors. Reviewed submitted version of manuscript: all authors. Approved the final version of the manuscript on behalf of all authors: Lawton. Study supervision: Lawton, Baranoski.

\section{Correspondence}

Michael T. Lawton: c/o Neuroscience Publications, Barrow Neurological Institute, St. Joseph's Hospital and Medical Center, Phoenix, AZ.neuropub@barrowneuro.org. 